\title{
Students' strategies for thematic map analysis
}

\author{
Lenka Havelkováa $^{\mathrm{a}}$ *, Martin Hanus ${ }^{\mathrm{a}}$, David Trokšiar ${ }^{\mathrm{a}}$ \\ ${ }^{a}$ Charles University, Faculty of Science, Centre for Geographical and Environmental Education, l.havelkova@ natur.cuni.cz, \\ martin.hanus@natur.cuni.cz,david.troksiar@natur.cuni.cz \\ * Corresponding author
}

Keywords: Strategy, Eye tracking, Thematic map, User study, Cartographic education

\begin{abstract}
:
When students use thematic maps during school lessons, how they are required to do so can substantially differ from the usual interaction with these maps in daily lives. Rather than quickly overview a map content as a whole or search for a particular object/value, they are challenged to solve specific problems or answer given tasks using a map. It is thus important to know not only how students use the map itself and which strategies they use when they are searching for a given object/value in the map face, but how they generally process during the map task solving. Given that, it is necessary to consider also how they get familiar with the presented task assignment, how and in which order they interconnect the information in the assignment with the information in the map elements, and possibly with the offered task answers. Moreover, to fully use the potential of thematic maps as educational tools, students should identify and compare spatial/temporal patterns of depicted phenomena, formulate predictions, generalizations, and conclusions based on the maps, and critically approach their content and design. Therefore, they should have developed efficient strategies for these cognitively more demanding map tasks.
\end{abstract}

For these reasons, the presented study focuses on strategies that students use when they are solving tasks requiring analysis of spatial patterns and spatial relations on thematic maps. Even though there are various methodological approaches for investigating individuals' strategies, it is the eye-tracking that is becoming more and more frequently used as it can directly identify the strategies and thus it avoids the subjectivity and incompleteness of participants' selfreported strategies. Our study combines the eye-tracking technology with a follow-up questionnaire to also understand the reasoning behind students' strategy choice.

For the eye-tracking experiment, a piloted version of the achievement test, consisting of 12 tasks, was used. Each task was presented as an individual stimulus together with the main map elements (i.e., map face, legend, graphic scale, and north arrow), and three possible answers (one correct answer and two distractors; see Figure 1). The widely used mapping methods in Czech school atlases and geography textbooks were chosen, namely area-shading, line symbols, choropleth mapping, and diagram mapping. Different mapping methods were used to test if and, if yes how, the chosen mapping methods influences students' strategy. All other characteristics of map design and layout were homogenized. The created maps represent a fictional area and data to avoid the potential influence of students' previous knowledge about depicted phenomena and familiarity with the area. In addition, the specific task demand was manipulated to investigate its impact on the strategy. Nevertheless, the tasks differ only in the means used during the analysis of the phenomena spatial distribution. The first tasks for each given mapping method do not require any special means. The second tasks are focused on analysing the distances, i.e., using a map scale. The third tasks are focused on using the cardinal points for the description of the spatial distribution.

A total of 53 students from gymnasia (i.e., selective secondary schools) in Czechia participated in the present study. All participating students were in the penultimate (3rd) or last (4th) year (i.e., 17-19 years of age) and had finished their mandatory geography education. Given that, their level of map skills and strategies used for map task-solving should be the highest possible developed by Czech upper secondary education.

\begin{tabular}{|c|c|c|}
\hline type & representative strategy & type description \\
\hline TMA & Task » Map » Answers & $\begin{array}{l}\text { get familiar with the task } » \text { solve the task using information gathered from the map } \\
\text { element }(\mathrm{s}) » \text { compare the solution with the given possible answers }\end{array}$ \\
\hline TAM & Task » Answers » Map & $\begin{array}{l}\text { get familiar with the task » check the given possible answers " solve the task using the } \\
\text { information from the map element(s), i.e., find which given answer is correct }\end{array}$ \\
\hline TxAx & Task » Map » Answers » Map & $\begin{array}{l}\text { get familiar with the task » start to solve the task using the map element(s) » check the } \\
\text { given possible answers » finish the task solving using (an)other map element(s) }\end{array}$ \\
\hline TM & Task » Map & $\begin{array}{l}\text { get familiar with the task » solve the task using information gathered from the map } \\
\text { element(s) without working with the given possible answers }\end{array}$ \\
\hline
\end{tabular}

Table 1. Theoretically set strategy types and their description. Note: The term "Map" in the representative strategy stands for all basic map elements. 
To identify participants' strategies, the collected eye-tracking data were analyzed using the GazeReplay method, i.e., by analyzing the video recordings of participants' eye movements. Based on detailed and repeated analysis of the recordings, both authors independently coded the strategies that participants used when they were solving the tasks. During the coding, the used strategies were assigned to the theoretical strategies that were previously set based on a cognitive walk-through with experts and the framework of Svenson's differentiation and consolidation theory of decision-making. The theoretically set strategies were categorized into four strategy types; see Table 1 for their brief description and Figure 1 for a simplified visualisation of sample strategies from one of the strategy types.

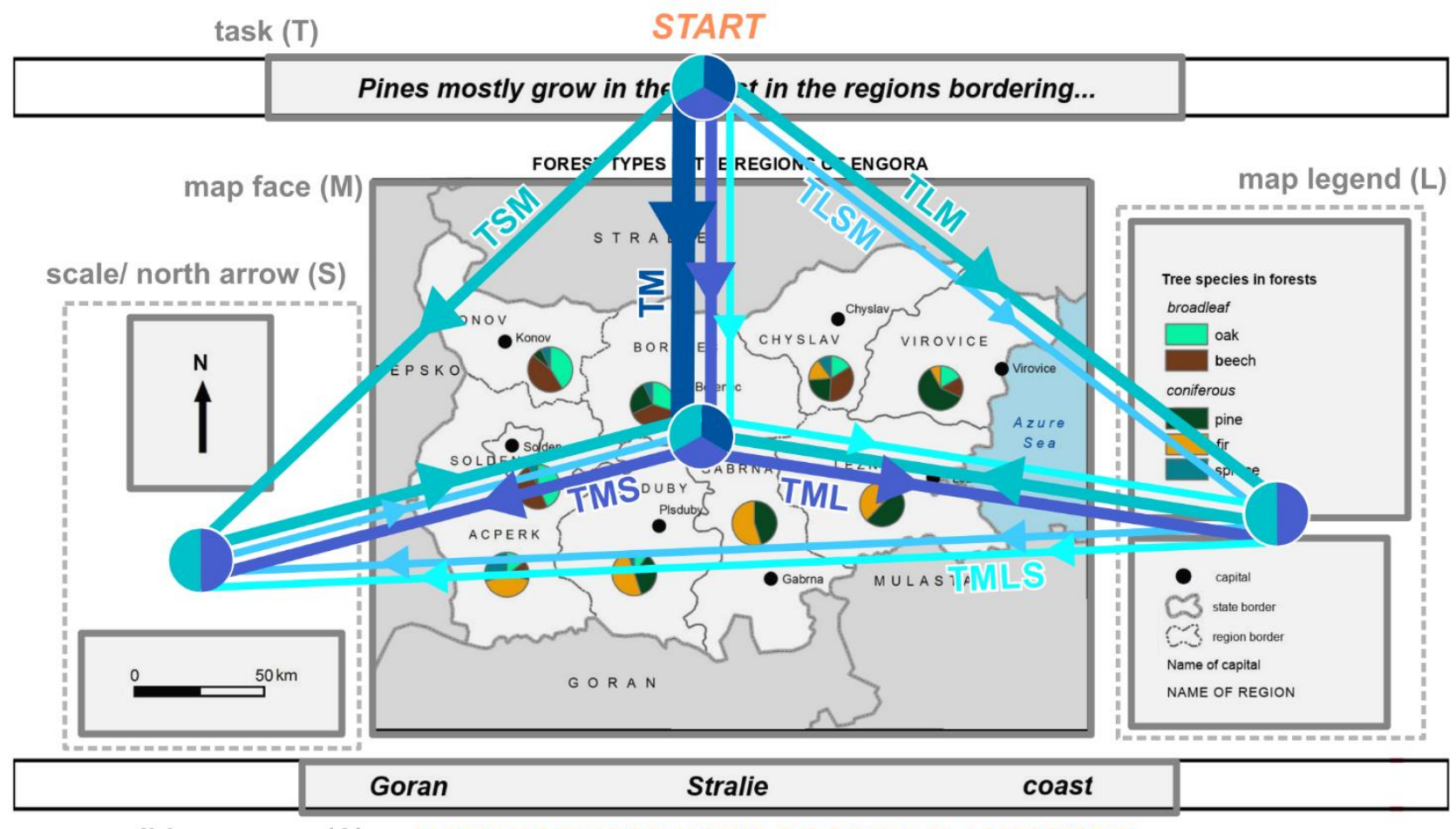

possible answers (A) NOT WORKING WITH POSSIBLE ANSWERS

Figure 1. Illustrative strategies from one of the four strategy types - TM strategy type.

The results of our study show that the participating upper-secondary students had on average developed equally broad repertoires of strategies for solving tasks focused on the analysis of thematic maps as previously investigated university students and cartographers. Similarly to them, the upper-secondary students most frequently processed the task-solving in a way in which they first got familiar with the task assignment, then started to solve the task using the map face and other map elements (largely the map legend), and only after that verified if their found solution matched the provided possible answers (i.e., they chose strategies from TMA type).

The tested students properly adapted their choice of strategy to specific task demands. Contrary to the task demand, they only marginally, if at all, changed their strategy due to the different mapping methods used. Since the students in almost all the cases paid attention only to the elements containing relevant information for the given task and were not substantially influenced by the map design, their strategies can be considered expert-like. In addition, students' statements in the follow-up questionnaire confirm the learning effect identified from the eye-tracking data. The students stated that they did not considerably change their approach to task-solving but gradually got used to the task structure, learned how to better orient in the map face, spent less time getting familiar with the map legend, were more cautious during the first task assignment reading, etc., and for these reasons were more efficient in task-solving.

Despite the relatively high students' success rate and efficiency of the strategies they chose, it is possible to identify several potentially problematic aspects of the used strategies and a few cognitive bottlenecks during the analysis of thematic maps. Based on them and the most efficient task-solving process of the students, it is possible to suggest how students' strategies for task-solving with maps can be appropriately developed from primary education, and possibly, how the cartographic production for schools can be enhanced. For instance, one of the specific practical outcomes based on the results of this study (and other following studies) could be a web-based adaptive application that would help teachers to develop a broad repertoire of efficient strategies for map use in their students and would pay special attention to the identified problematic aspects of commonly used strategies and to individuals' needs and level. 